\title{
Numerical solution of free-boundary problems in fluid mechanics. Part 3. Bubble deformation in an axisymmetric straining flow
}

\author{
By G. RYSKIN† AND L. G. LEAL \\ Department of Chemical Engineering, California Institute of Technology, \\ Pasadena, California 91125
}

(Received 11 April 1983 and in revised form 27 April 1984)

We consider the deformation of a bubble in a uniaxial extensional flow for Reynolds numbers in the range $0.1 \leqslant R \leqslant 100$. The computations show that the bubble bursts at a relatively early stage of deformation for $R \geqslant O(10)$, never reaching the highly elongated shapes observed and predicted at lower Reynolds numbers. We also compute the deformation of the bubble under the assumption of potential flow, and conclude that the potential-flow solution provides a good approximation to the real flow in this case for $R \geqslant O(100)$.

\section{Introduction}

The present paper is the third in a series (see Ryskin \& Leal 1984 $a, b$, hereinafter denoted as Parts 1 and 2 ) in which we use numerical methods to study the deformation of a bubble in axisymmetric flow fields at finite Reynolds number. Here we consider uniaxial extensional flow. Previous theoretical studies of this problem have been restricted to either zero-Reynolds-number flows (Taylor 1934, 1964; Buckmaster 1972; Barthes-Biesel \& Acrivos 1973; Youngren \& Acrivos 1976) or potential flow (Miksis 1981), with the exception of the non-zero Reynolds-number, slender-body analysis of Acrivos \& Lo (1978), which merely requires that the bubble be slender. Although laboratory studies have generally been restricted to highly viscous fluids (and to two-dimensional, rather than uniaxial, extension), many practical flows involve low-viscosity fluids such as water, and the Reynolds numbers in these systems easily attain values $O\left(10^{2}-10^{3}\right)$. For example, to achieve $R=100$ in water with a bubble of $1 \mathrm{~cm}$ diameter requires a strain rate of only $O\left(1 \mathrm{~s}^{-1}\right)$. The present study covers $R$ ranging from 0.1 to 100 , as well as potential-flow results, in all of which the deformation may become quite large.

At low Reynolds number both experiments and theory (Taylor 1964) show that a bubble may become extremely elongated and develop pointed ends where the curvature tends locally to extremely large values. Such cases are not accessible with our present numerical technique, and the slender-body analysis of Taylor (1964), Buckmaster (1972) and Acrivos \& Lo (1978) cannot be checked now. However, we can compare our results in the low-Reynolds-number range $R=0.1$ and 1 with the predictions of Youngren \& Acrivos (1976), who used the boundary-integral technique for creeping flow to consider large deformations of a bubble in extensional flow at

† Present address: Department of Chemical Engineering, Northwestern University, Evanston, Illinois 60201 . 
zero Reynolds number. We can also compare our results in the potential-flow limit $(R \rightarrow \infty)$ with the solution of Miksis (1981).

Our results for intermediate Reynolds numbers $R=10$ and 100 appear to us to be of greater interest. These results indicate that the presence of inertia leads to breakup of a bubble at a quite early stage of deformation.

\section{Statement of the problem}

We consider an incompressible gas bubble of volume $\frac{4}{3} \pi a^{3}$ subjected to a steady uniaxial extensional flow of a fluid with constant density $\rho$ and constant viscosity $\mu$. The density and viscosity of the gas inside the bubble are assumed to be negligible in comparison with those of the liquid. Furthermore, the surface of the bubble is assumed to be characterized completely by a uniform surface tension $\gamma$. Finally, we neglect all effects of gravity including the hydrostatic pressure variation in the fiuid. The latter condition has been adopted in all previous studies of the problem, and is necessary in order that the shape be axisymmetric and exhibit fore-aft symmetry. If the $x$-axis of cylindrical coordinates $(x, \sigma, \phi)$ is directed along the axis of symmetry, the (dimensional) velocity field far from the bubble is given by

$$
\boldsymbol{u}=\boldsymbol{E} \cdot \boldsymbol{r}, \quad \boldsymbol{E}=\boldsymbol{E}\left(\begin{array}{rrr}
1 & 0 & 0 \\
0 & -\frac{1}{2} & 0 \\
0 & 0 & -\frac{1}{2}
\end{array}\right), \quad E>0,
$$

where $E$ is the principal strain rate. We use the equivalent radius $a$ of the bubble as a characteristic lengthscale and the product $E a$ as a characteristic velocity scale. The numerical solution is computed on a boundary-fitted curvilinear orthogonal coordinate system $(\xi, \eta, \phi)$ obtained by the technique of orthogonal mapping using the algorithm described in Part 1 . All necessary equations and boundary conditions were also given in Part 1, with the exception of the expression for the pressure at the bubble surface. In the present work, the latter was calculated using equation (3) of Part 2, but with the hydrostatic pressure contribution set equal to zero. As in the case of Part 2, all results reported here were obtained on a $40 \times 40$ grid in the $(\xi, \eta)$-coordinates. The relevant dimensionless parameters are the Reynolds number $R \equiv 2 \rho(E a) a / \mu$ based on the equivalent diameter $2 a$ of the bubble, and the Weber number $W \equiv 2 \rho(E a)^{2} a / \gamma$.

\section{Numerical results and discussion}

We have done computations for $R=0.1,1,10,100$ and $\infty$ (potential flow), gradually increasing $W$ in each case until a value was reached beyond which a solution could not be obtained. At low Reynolds numbers $(R=0.1$ and 1$)$ the tips of the bubble become increasingly pointed with increase of $W$, and the limit on $W$ that we could achieve is a consequence of our inability to resolve these regions with a $40 \times 40$ grid in the $(\xi, \eta)$-plane. At the higher Reynolds numbers, on the other hand, we believe that our inability to obtain steady solutions beyond a certain $W$ is a result of the fact that steady axisymmetric solutions do not exist for larger values of $W$, i.e. for $W>W_{\mathrm{c}}(R)$, where $W_{\mathrm{c}}(R)$ denotes a 'critical' value for breakup. We shall discuss both cases in detail in the remainder of this section. The computed bubble shapes are shown in figure 1.

At low Reynolds numbers there is a trend, also predicted by the creeping-flow theory and observed in experiments (see Acrivos \& Lo 1978, and references therein) for a bubble to elongate monotonically with increase in the capillary number 
$R$

0.1

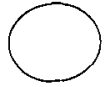

$W=0.005$

1

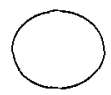

0.05

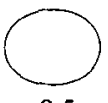

0.5

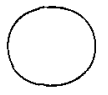

1.0

$\infty$

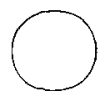

1.0

100

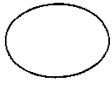

0.01

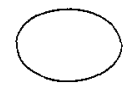

0.1

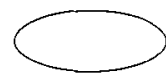

0.02

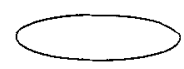

0.2
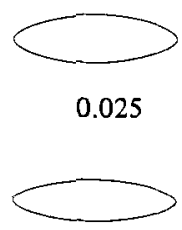

0.25

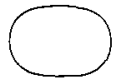

2.0

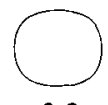

2.0

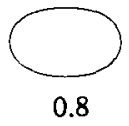

0.8

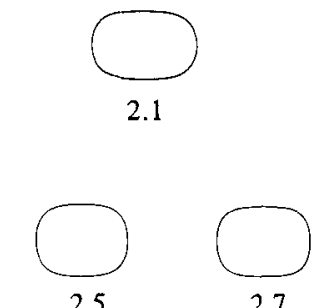

2.7

Figure 1. Shapes of a bubble in uniaxial extensional flow as a function of Reynolds and Weber numbers; $R=\infty$ corresponds to the potential-flow solution.

$\mu E a / \gamma=W / R$. In the process of elongation, the curvature of the surface becomes very large at the tips of the bubble. The largest values of $W$ shown in figure 1 for $R=0.1$ and 1 represent the limit of our ability to resolve this region of high curvature with the $(\xi, \eta)$-coordinate system that was used and a $40 \times 40$ grid, given the necessity for reasonable rates of convergence. There is no reason to believe that steady axisymmetric solutions could not have been achieved at larger Weber numbers if more grid points were used, or possibly the $(\xi, \eta)$-coordinates redefined to concentrate a greater proportion of coordinate lines in the vicinity of the bubble tips. It is noteworthy that the slender-body theory at $R=0$ due to Taylor (1964), Buckmaster (1972) and Acrivos \& Lo (1978) predicts no breakup, while the low-Reynolds-number analysis of Acrivos \& Lo (1978) predicts breakup at higher $W$ than was achieved here. To demonstrate this latter point it is necessary to convert the breakup criterion of Acrivos \& Lo (1978) from its original form

$$
\frac{\mu E a}{\gamma}\left(\frac{\rho a \gamma}{\mu^{2}}\right)^{\frac{1}{5}}=0.284
$$

in terms of the capillary number $\mu E a / \gamma$ to one that is explicit in Weber number : either

$$
W_{\mathrm{c}}=0.247 R^{3}
$$

or

$$
W_{\mathrm{c}}=0.162 \alpha^{\frac{3}{5}}, \quad \alpha \equiv \frac{\rho a \gamma}{\mu^{2}} .
$$




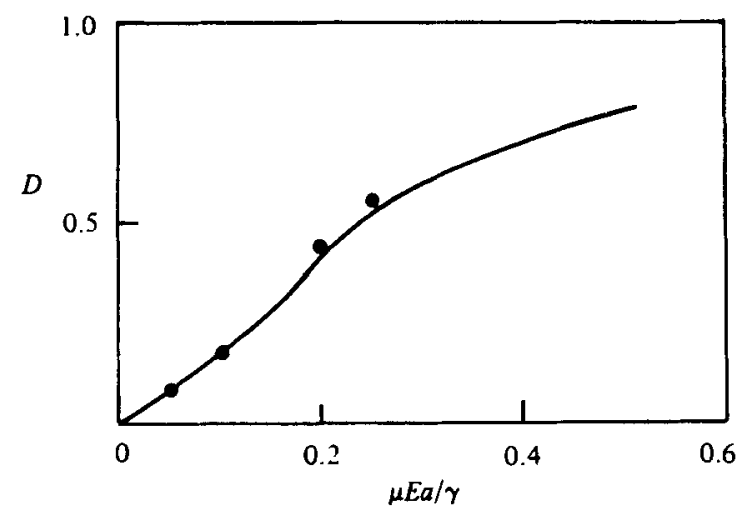

Figure 2. Comparison of the present results for $R=0.1$ (points) with the results of Youngren $\&$ Acrivos (1976) for $R=0$ (solid line, plotted in terms of the deformation parameter $D \equiv(l-b) /(l+b)$ as a function of the capillary number $\mu E a / \gamma$. Here $l$ and $b$ are respectively the half-lengths of the major and minor axes of the bubble profile.

The latter is slightly preferable, because it gives a specific prediction for the critical $W$ in terms solely of material (rather than dynamic) parameters. For $R=0.1$, where Acrivos \& Lo's theory may be expected to apply, $W_{\mathrm{c}}$ is predicted to be 0.044 , which is considerably in excess of the value 0.025 that was reached numerically. At $R=1$ the Acrivos-Lo criterion gives $W_{\mathrm{c}}=0.247$, but the apparent agreement with the largest value 0.25 achieved numerically is almost certainly fortuitous since the analy tic result is expected to hold only for $R e \ll 1$. $\dagger$

Our steady-state solutions for $R=0.1$ are in good agreement with the creeping-flow solutions of Youngren \& Acrivos (1976), which were obtained using the boundaryintegral technique. A comparison of the solutions in terms of $D \equiv(l-b) /(l+b)$, where $l$ is the bubble half-length and $b$ its radius at the midsection, versus $\mu E a / \gamma$ is shown in figure 2 . It can be seen that our solution predicts a slightly higher deformation for $R=0.1$ than Youngren \& Acrivos' solution for $R=0$. The difference is presumably due to the weak effect of inertia. The slender-body analysis of Acrivos \& Lo also shows slightly increased deformation upon the inclusion of inertia. Our results for $R=1$ show the same general 'low-Reynolds-number pattern' of deformation, with the bubble becoming strongly elongated prior to breakup. The degree of deformation for any $\mu E a / \gamma$ is increased relative to the solutions for $R=0$ and 0.1 .

A qualitative change in the nature of the deformation takes place at some Reynolds number between 1 and 10 . At $R=10$ and 100 the bubble does not become elongated. As can be seen from figure 1, the bubble first assumes the shape of an oval, but with

+ The reformulation of Acrivos \& Lo's criterion for bubble breakup in terms of $W$ would appear to be more natural than the original form in terms of capillary number because bubble breakup is solely inertial in origin, unlike bubble elongation, which depends (for $R<O(1)$ ) on the balance between surface-tension and viscous forces, and is therefore determined by the capillary number. The only surprising fact is that $W_{c}$ does not appear as a constant, as would be expected, but rather as a function of $\alpha$. However, this is because $W$ is based on the equivalent diameter $2 a$ of the undeformed bubble, which does not represent a true characteristic lengthscale of the problem when the bubble is strongly elongated. A more appropriate definition of Weber number in this case is $W^{*}=2 \rho(E l)^{2} /(\gamma / b)$, where the characteristic velocity $E l$ is proportional to the bubble half-length $l$, and the capillary force is inversely proportional to $b$, the bubble midsection radius. At the point of breakup, Acrivos \& Lo found for a strongly elongated bubble $l / a=2.37 \alpha^{-\frac{2}{3}}$ and $b / l=0.295 \alpha^{\frac{3}{5}}$. Thus $W_{\mathrm{c}}^{*}=0.636$ at breakup. This form of Acrivos $\&$ Lo's criterion shows most clearly the inertial nature of bubble breakup, even for $R<O(1)$. 
further increase of $W$ the side surface approaches a circular cylindrical shape. Our attempts to obtain a converged solution for $R=10$ and $W=1$ and for $R=100$ and $W=2.2$ invariably failed, resulting in fast divergence, even though solutions for the same Reynolds numbers and $W$ less by only 0.1 were readily obtained (see figure 1 ). In these cases there are no surface points where the curvature is much higher than $O(1)$, and so it is unlikely that purely numerical difficulties could be a source of divergence. Monitoring the shape of the bubble during an attempt to compute these diverging solutions, we observed that after some number of iterations the side surface of the bubble became cylindrical at the equator (i.e. $\kappa_{(\eta)}$ became zero there), then a 'waist' appeared and divergence followed. Though our numerical procedure in its present form does not simulate a real transient (unsteady) flow, it is intuitively clear from the sequence of shapes for $R=10$ and 100 in figure 1 that further increase in Weber number would lead to appearance of the 'waist' in a real flow. Again intuition suggests that a bubble with a waist is unlikely to be stable and should burst into two parts (indeed, such is the case for a slender bubble with a waist at $R=0$, as shown by Hinch 1980). Accordingly, we conclude that bubble breakup (or, more accurately, the onset of transient elongation) occurs for $0.9<W_{\mathrm{c}}<1$ at $R=10$, and $2.1<W_{\mathrm{c}}<2.2$ at $R=100$. Although a closer estimate of the critical Weber number could have been obtained by looking for solutions within the above intervals, this was not done in present work. Obviously, the bounds on $W_{\mathrm{c}}$ that we have obtained are accurate within a few percent.

We do not present the streamline plots corresponding to the shapes in figure 1, since there is no flow separation, and accordingly the streamlines provide little additional insight. It is quite unlikely that separation will appear at higher Reynolds numbers since the only source of vorticity is boundary curvature, which seems to be approaching an $O(1)$ limit as $R$ is increased for any fixed $W$ (see Part 2 for a discussion of the connection between vorticity production and separation). Also, the velocity and pressure fields in an extensional flow differ from those in the uniform flow in a way that makes flow separation much less likely, e.g. there is no separation in an extensional flow past a solid sphere at Reynolds numbers up to $O\left(10^{3}\right)$ (see Ryskin $1980, \S 7$ ). If separation does not occur, a solution under the assumption of irrotational flow should provide a good approximation to the real flow for $R \rightarrow \infty$. Such solutions have been computed by Miksis (1981) using the boundary-integral technique, and it is of interest to compare his results with corresponding results from the very different approach taken here. Accordingly, we have computed inviseid irrotational solutions by setting the vorticity equal to zero everywhere and neglecting those terms that include a factor $1 / R$ in the expression for normal stress, which thus becomes

$$
\tau_{\xi \xi}=-p_{\text {dyn }}=u_{\eta}^{2}
$$

Application of the present method becomes quite easy in this case, and takes only about $5-10 \mathrm{~min}$ of CPU time on the VAX-11/780. The results presented in figure 1 show that the trend that is already apparent at $R=10$ continues to $R \rightarrow \infty$. The Weber number $W=2.7$ is the highest (in steps of 0.1 ) for which a converged solution could be obtained in the potential-flow limit; for $W=2.8$ the 'waist' appears at some stage of the iteration process and then keeps contracting without converging to an equilibrium solution. We may therefore conclude that the critical Weber number for bubble breakup in the limit $R \rightarrow \infty$ is between 2.7 and 2.8, i.e. $2.7<W_{\mathrm{c}}<2.8$. Miksis (1981) predicts (in our notation) $W_{\mathrm{c}}=2.76$. The agreement is good and supports our interpretation of the appearance of the 'waist' and subsequent divergence of our numerical scheme as signifying bubble breakup. 
An approximate interpolation formula for the critical Weber number that fits the low- $R$ asymptote of Acrivos \& Lo (1978), as well as the approximate critical values $W_{\mathrm{c}}=0.95,2.15$ and 2.76 from the present work (and Miksis 1981) at $R=10,100$ and $\infty$, is

$$
\left(\frac{1}{W_{\mathrm{c}}}\right)^{\frac{10}{9}}=\left(\frac{1}{2.76}\right)^{\frac{10}{9}}+\left(\frac{1}{0.247 R^{\frac{3}{3}}}\right)^{\frac{10}{9}}
$$

This empirical interpolation formula can also be expressed in an implicit form in terms that depend only on bubble size and liquid properties:

$$
W_{\mathrm{c}}^{\frac{10}{8}}+10.9 \alpha^{-\frac{5}{12}} W_{\mathrm{c}}^{\frac{25}{36}}-3.09=0 .
$$

We currently know of no experimental data that could be used to corroborate this result.

It should be emphasized that the above results are valid only for bubbles (whose density is negligible) but not for inviscid drops. These two cases are identical only if the Reynolds number is zero or if the shape is fixed (spherical). Otherwise, one must take into account the variation of pressure inside the inviscid drop. In particular, if the drop is neutrally buoyant, the $u_{\eta}^{2}$ term in the expression for normal stress (see Part 2, equation (3)) will be exactly cancelled by a similar term inside the drop. This $u_{\eta}^{2}$ term is the dominant contribution to the normal stress, and thus to the shapes of bubbles, at high $R$. In particular, the strong deformation at the equator of the bubble for $R>O(10)$ (and, eventually, the development of the 'waist' and subsequent bursting of the bubble) is apparently due to the high stagnation pressure at this point. The complete cancellation of the $u_{\eta}^{2}$ term in the case of a neutrally buoyant (viscous or inviscid) drop is certain to have a profound effect on the deformation. We note in passing that the above remarks are also applicable to Acrivos \& Lo's (1978) solution at non-zero Reynolds number in the slender-body regime, and to Miksis' (1981) solution at $R \rightarrow \infty$. These solutions are valid only for a bubble, and not for an inviscid drop as suggested by the authors.

\section{Comparison with rising bubble}

In a certain sense, the deformation of a bubble in an extensional flow is a much simpler phenomenon than the deformation of a rising bubble in a quiescent fluid. In particular, the surface values of the normal stress, the hydrostatic pressure and the dynamic pressure balance exactly in an integral sense in the case of a rising bubble (see Part 2) since the resultant force on the bubble is zero. Thus deformation in this case is a consequence of local differences in stress and pressure distributions, which are extremely difficult to anticipate, balanced against capillary forces. On the other hand, the balance of forces to achieve zero net force in the extensional flow is satisfied owing entirely to the fore-aft symmetry of the bubble. Thus one or the other of the pressure and normal-stress contributions can become dominant over the others, and then deformation occurs as a result of this one distribution balanced against capillary forces. Because of this, the qualitative mode of deformation is much more accessible to intuition in the latter case. For example, the tendency for a bubble in extensional flow at low Reynolds number to extend in the direction of the principal axis of strain is easily anticipated from the viscous-stress and pressure distribution at the surface of a spherical bubble in the same flow. Similarly, at high Reynolds number, the dynamic pressure is the dominant contribution to the stress balance at the bubble surface, and it is evident that the bubble should be pushed inward at all stagnation ponts owing to the higher pressure there, as was indeed observed in the computations. 
The above discussion also clarifies the difference between the two problems in terms of the importance of the dimensionless groups for estimating the degree of deformation. In the most general sense (i.e. not referring to any particular problem), one would expect the degree of deformation at low Reynolds number to depend upon the capillary number $W / R$, which is a measure of the magnitude of viscous stresses relative to capillary forces. For example, when $W / R=O(1)$ one should normally expect large deformation (and possibly even breakup) on the basis of the orderof-magnitude estimate of viscous 'deforming' forces relative to the surface-tension 'restoring' forces that is inherent in $W / R$. The Weber number would be expected to play a similar role at higher $R$, since it is a measure of the order of magnitude of dynamic pressure forces relative to capillary forces. These expectations are generally borne out in the case of an extensional flow, as can be seen from the results of figure 1. However, in the case of a rising bubble, these expectations prove completely wrong. The capillary number does not play a role at all. Furthermore, though the Weber number does determine the deformation at non-zero Reynolds numbers, the deformation is rather small at $W=O(1)$, and, indeed, extremely high Weber numbers can be reached experimentally for spherical-cap bubbles without breakup, the shape of a bubble becoming essentially independent of $W$ above some value of order 15-20 (see Part 2). Unlike the extensional-flow problem where $W / R$ and $W$ do provide a direct measure of the strength of the dominant deforming forces relative to the restoring tendency of surface tension, the overall 'integral' balance between static and dynamic pressures and the viscous normal stress in the case of a rising bubble means that no single one of these deforming forces can become dominant at any $W / R$ or $W$.

This work was supported by a grant from the Fluid Mechanics Program of the National Science Foundation.

\section{REFERENCES}

Acrivos, A. \& Lo, T. S. 1978 Deformation and breakup of a single slender drop in an extensional flow. J. Fluid Mech. 86, 641-672.

Barthes-Biesel, D. \& Acrivos, A. 1973 Deformation and burst of a liquid droplet freely suspended in a linear shear field. J. Fluid Mech. 61, 1-21.

Buckmaster, J. D. 1972 Pointed bubbles in slow viscous flow. J. Fluid Mech. 55, 385-400.

Hinch, E. J. 1980 The evolution of slender inviscid drops in an axisymmetric straining flow. $J$. Fluid Mech. 101, 545-553.

Miksis, M. J. 1981 A bubble in an axially symmetric shear flow. Phys. Fluids 24, 1229-1231.

Ryskin, G. 1980 The extensional viscosity of a dilute suspension of spherical particles at intermediate microscale Reynolds numbers. J. Fluid Mech. 99, 513-529.

Ryskin, G. \& LEAL, L. G. $1984 a$ Numerical solutions of free-boundary problems in fluid mechanics. Part 1. The finite-difference technique. J. Fluid Mech. 148, 1-17.

Ryskin, G. \& LEAL, L. G. $1984 b$ Numerical solutions of free-boundary problems in fluid mechanics. Part 2. Buoyancy-driven motion of a gas bubble through a quiescent liquid. J. Fluid Mech. 148, 19-35.

TAYLOR, G. I. 1934 The formation of emulsions in definable fields of flow. Proc. R. Soc. Lond. A 146, 501-523.

TAYLOR, G. I. 1964 Conical free surfaces and fluid interfaces. In Proc. 11th Intl Congr. Appl. Mech., Munich (ed. H. Görtler), pp. 790-796.

YoUNGREN, G. K. \& ACRIvos, A. 1976 On the shape of a gas bubble in a viscous extensional flow. J. Fluid Mech. 76, 433-442. 